\title{
Post-mortem cardiac magnetic resonance parameters in normal and diseased conditions
}

\author{
Giuseppe Femia ${ }^{1}$, Neil Langlois ${ }^{2,3}$, Jim Raleigh ${ }^{4}$, Sunthara Rajan Perumal ${ }^{5}$, Christopher Semsarian ${ }^{1,6,7}$, \\ Rajesh Puranik ${ }^{1,6}$
}

${ }^{1}$ Sydney Medical School, Faculty of Medicine and Health, The University of Sydney, Camperdown, NSW, Australia; ${ }^{2}$ Forensic Science South Australia, Adelaide, South Australia, Australia; ${ }^{3}$ School of Medical and Health Sciences, University of Adelaide, Adelaide, South Australia, Australia; ${ }^{4}$ Department of Radiology, Royal Prince Alfred Hospital, Camperdown, NSW, Australia; ${ }^{5}$ South Australia Health \& Medical Research Institute, Preclinical, Imaging \& Research Laboratories, Adelaide, Australia; ${ }^{6}$ Department of Cardiology, Royal Prince Alfred Hospital, Camperdown, NSW, Australia; ${ }^{7}$ Agnes Ginges Centre for Molecular Cardiology Centenary Institute, The University of Sydney, Camperdown, NSW, Australia

Contributions: (I) Conception and design: G Femia, N Langlois, R Puranik; (II) Administrative support: G Femia, N Langlois, SR Perumal, Semsarian, R Puranik; (III) Provision of study materials or patients: G Femia, N Langlois, J Raleigh, SR Perumal, R Puranik; (IV) Collection and assembly of data: G Femia, N Langlois, SR Perumal, R Puranik; (V) Data analysis and interpretation: All authors; (VI) Manuscript writing: All authors; (VII) Final approval of manuscript: All authors.

Correspondence to: Dr. Giuseppe Femia. Sydney Medical School, Faculty of Medicine and Health, The University of Sydney, Camperdown, NSW, Australia. Email: femia82@gmail.com.

Background: Post-mortem cardiac magnetic resonance (CMR) is a non-invasive alternative to conventional autopsy. At present, diagnostic guidelines for cardiovascular conditions such as hypertrophic cardiomyopathy have not been established. We correlated post-mortem CMR images to definite conventional autopsy findings and hypothesed that elevated T2-weighted signal intensity and RV to LV area ratios can identify myocardial infarction and pulmonary emboli respectively.

Methods: For this unblinded pilot sub-study, we selected cases from the original blinded study that compared post-mortem imaging to conventional autopsy in patients referred for coronial investigation between October 2014 to November 2016. Three groups of scans were selected based on the cause of death identified by conventional autopsy: non-cardiovascular causes of death with no structural cardiac abnormality i.e., control cases, acute/subacute myocardial infarction and pulmonary emboli. Left ventricular (LV) wall thickness, LV myocardial signal intensity and ventricular cavity areas were measured.

Results: Fifty-six scans were selected [39 (69.6\%) males]: 37 (66.1\%) controls, eight (14.3\%) acute/ subacute myocardial infarction and eleven (19.6\%) pulmonary emboli. The median age was 61 years [Interquartile range (IQR) 50-73] and the median time from death to imaging and autopsy was 2 days (IQR $2-3)$ and 3 days (IQR 3-4). The septal and lateral walls were thicker $\{15 \mathrm{~mm} \mathrm{[13-17]} \mathrm{and} 15 \mathrm{~mm}$ [14-18]\} on post-mortem CMR than published ante-mortem measurements. Areas of acute/subacute myocardial infarction had significantly higher T2-weighted signal intensity (normalised to skeletal muscle) compared to normal myocardium in those who died from other causes $\{2.5[2.3-3.0$.] vs. $1.9[1.8-2.3] ; \mathrm{P}<0.001\}$. In cases with pulmonary emboli, there was definite RV enlargement with a larger indexed RV to LV area ratio compared to those who died from other causes $\{2.9[2.5-3.0]$ vs. $1.8[1.5-2.0] ; \mathrm{P}<0.001\}$.

Conclusions: We present potential post-mortem CMR parameters to identify important cardiovascular abnormalities that may be beneficial when conventional autopsy cannot be performed. In patients without cardiovascular disease, $\mathrm{LV}$ wall thickness was found to be unreliable in diagnosing hypertrophic cardiomyopathy without histological and/or genetic testing. Elevated T2 signal intensity and RV to LV area ratios may be useful markers for acute/subacute myocardial infarction and pulmonary emboli. Larger studies will be necessary to define cut-offs.

Keywords: Post-mortem cardiac magnetic resonance; conventional autopsy; myocardial infarction; pulmonary emboli; hypertrophic cardiomyopathy 
Submitted Nov 22, 2020. Accepted for publication Feb 15, 2021.

doi: $10.21037 /$ cdt-20-948

View this article at: http://dx.doi.org/10.21037/cdt-20-948

\section{Introduction}

Conventional autopsy is considered the 'gold standard' for post-mortem examination but due to declines in referrals and an increasing need for a non-invasive alternative, postmortem cardiac magnetic resonance (CMR) has become a popular option $(1,2)$. Post-mortem CMR has been shown to be helpful in identifying some cardiovascular abnormalities and the cause of death without the need for an invasive examination (3-5). In one study of patients younger than 35 years of age, dedicated post-mortem MR imaging of the heart correctly identified the diagnosis of all patients with cardiovascular abnormalities on conventional autopsy (3). Another study subsequently found that when the radiologist was confident about the cause of death, $40 \%$ of the cases referred for coronial investigation would not require a conventional autopsy (6). Despite these previous results, CMR has been found to be less accurate than conventional autopsy for identifying common cardiovascular abnormalities such as hypertrophic cardiomyopathy (HCM), acute/subacute myocardial infarction and pulmonary emboli (7). It remains common practice to use ante-mortem criteria to diagnose pathological conditions despite significant post-mortem changes to the structure and composition of organs. Moving forward, it will be important to develop dedicated postmortem CMR guidelines to differentiate normal structures from pathological conditions.

In this unblinded pilot study, we correlated post-mortem CMR images to the definite findings of conventional autopsy and aimed to identify diagnostic parameters. We hypothesised that elevated T2-weighted signal intensity and $\mathrm{RV}$ to $\mathrm{LV}$ area ratio measurements can identify myocardial infarction and pulmonary emboli respectively. We present the following article in accordance with the STROBE reporting checklist (available at: http://dx.doi.org/10.21037/ cdt-20-948).

\section{Methods}

The study was conducted in accordance with the Declaration of Helsinki (as revised in 2013). The State Coroner for South Australia approved a joint initiative between Forensic Science South Australia and the
South Australian Health and Medical Research Institute (SAHMRI) and performed in accordance with NHMRC human ethics guidelines. Informed consent was taken from all the patients.

\section{Study design and patient population}

In this retrospective study, CMR scans were selected from our original blinded study (7), where we evaluated the accuracy of post-mortem CMR in identifying causes of death as reported by autopsy between October 2014 to November 2016. For the current study, the same investigators selected cases reported to have either acute or subacute myocardial infarction, pulmonary emboli or no structural cardiac abnormality (i.e., control cases) on autopsy. The investigators than re-evaluated the CMR scans for myocardial wall thickness, myocardial signal intensity and ventricular cavity area. At the time of autopsy, de-identified details of the death, some limited medical history from the police report and information obtained directly from family members were available. The results of previous investigations such as electrocardiography were not available.

\section{Cardiac magnetic resonance imaging}

MR imaging was performed on a Siemens Sonata $1.5 \mathrm{~T}$ system (Siemens Medical Solutions USA, Inc, Malvern, PA, USA). The deceased individuals were scanned in the supine position within two non-ferrous body bags. The following sequences were acquired: T2 short tau inversion recovery (STIR) sequence was acquired in multiple long axis cardiac planes; left ventricular long axis (LVLA), right ventricular long axis (RVLA), four-chamber, left ventricular outflow tract (LVOT) and right ventricular outflow tract (RVOT). In addition, T2 turbo spine echo (TSE) and T2 STIR sequences were acquired according to the cardiac axis in the short axis (8-12 slices) plane through the entire cardiac mass from base to apex. Image parameters: repetition time $=8 \mathrm{~ms}$; echo time $=500 \mathrm{~ms}$; flip angle $=90$ degrees; fractional shortening $=1.5 \mathrm{~ms}$; slice thickness $5.0 \mathrm{~mm}$; matrix $168 \times 184$; and temporal resolution $=40 \mathrm{~ms}$. Images were reviewed by two cardiologists (RP, Level III SCMR and GF-Level III 
SCMR).

\section{Cardiac magnetic imaging analysis}

All scans were transferred to a dedicated computer where myocardial wall thickness, myocardial signal intensity and ventricular areas were measured on the Horos workstation version 3.3.6. Left ventricular wall thickness measurements were taken at standard anatomical regions; septum, anterior, lateral and inferior walls from T2 short-axis images in the mid ventricular slice at the level of the papillary muscles. At each position, the average of three separate measurements were reported. Subacute myocardial infarction was diagnosed on conventional autopsy with the corresponding CMR region used to measure signal intensity. Signal intensity measurements for normal myocardium was performed at standard anatomical regions. Three region of interest (ROI) circles measuring $0.5 \mathrm{~cm}^{2}$ were manually contoured and standardised to skeletal muscle (right deltoid). The median value of the three measurements were reported. RV and $L V$ areas were measured by manually drawing endocardial borders on four-chamber images and indexed to body surface area; marked coagulation in the right atrium and ventricle made it difficult to recognise the endocardial border in short-axis views. All measurements were performed by both GF and RP.

\section{Conventional autopsy and definitions}

Conventional autopsy was performed in a single highvolume centre by an experienced pathologist (NL) as described previously (7). Cardiomegaly was assessed with reference to published ranges for post-mortem heart weight $(8,9)$. Left ventricular hypertrophy was diagnosed by the pathologist guided by published guidelines $(10,11)$. Acute and subacute myocardial infarction were defined by the presence of intracoronary thrombus and/or interstitial oedema, coagulative necrosis (pyknosis, karyorrhexis) or poly-mononuclear cell infiltration and macrophages, lymphocytes or scant collagen (12). Pulmonary emboli were defined as laminated and non-adherent clots.

\section{Reproducibility}

Inter-observer variability was assessed by comparing independent measurements by the primary operator (GF) and a second blinded CMR cardiologist (RP). The co-efficient of variation was determined by dividing the difference between the measurements by the mean measurement for the two operators. Agreement between the measurements and the methods was further analysed using the intra-class co-efficient as described by Shrout and Fleiss (13). The inter-observer reproducibility was assessed using intra-class correlation and Bland-Altman analysis by calculating the bias (mean difference) and the $95 \%$ limits of agreement (1.96 SD around the mean difference).

\section{Statistical analysis}

All values are given as median and interquartile range (IQR). A P value $<0.05$ was considered significant. Numerical data was performed using Microsoft Excel and Statistical Practice for the Social Sciences (SPSS) software (Version 21, Armonk, NY: IBM Corp). Gaussian-distribution was assessed using the Kolmogorov-Smirnov test with a $\mathrm{P}$ value $<0.05$ considered significant. Differences in continuous variables between the three groups were analysed using an independent $t$-test and differences in categorical variables were assessed using Fisher's exact probability test. The inter-observer reproducibility was assessed using intra-class correlation and Bland-Altman analysis by calculating the bias (mean difference) and the $95 \%$ limits of agreement (1.96 standard deviation around the mean difference). Receiver operator characteristics (ROC) curve analysis was used to determine the area under the curve and the optimal cutoff values of myocardial signal intensity and $\mathrm{RV}$ to $\mathrm{LV}$ area ratio. Comparison of the area under the ROC curves was performed using the method described by Hanley et al. (14).

\section{Results}

\section{Baseline patient characteristics}

Fifty-six post-mortem CMR scans were selected [39 (69.6\%) males] with a median age at death of 61 years [Interquartile range (IQR) 50-73] and a median time from death to imaging and autopsy of 2 (IQR 2-3) and 3 days (IQR $3-4)$, respectively. From conventional autopsy, twentynine $(51.8 \%)$ had a non-cardiovascular cause of death, eight $(14.3 \%)$ had no identifiable cause but no structural cardiovascular abnormality, eight $(14.3 \%)$ died from acute or subacute myocardial infarction and eleven (19.6\%) died from pulmonary thrombi. $36(64.3 \%)$ were found dead after an unwitnessed collapse and $20(35.7 \%)$ had a witnessed collapsed and died after unsuccessful resuscitation. Fortysix $(82.1 \%)$ died at their place of residence, four $(7.1 \%)$ died 
Table 1 Baseline characteristics

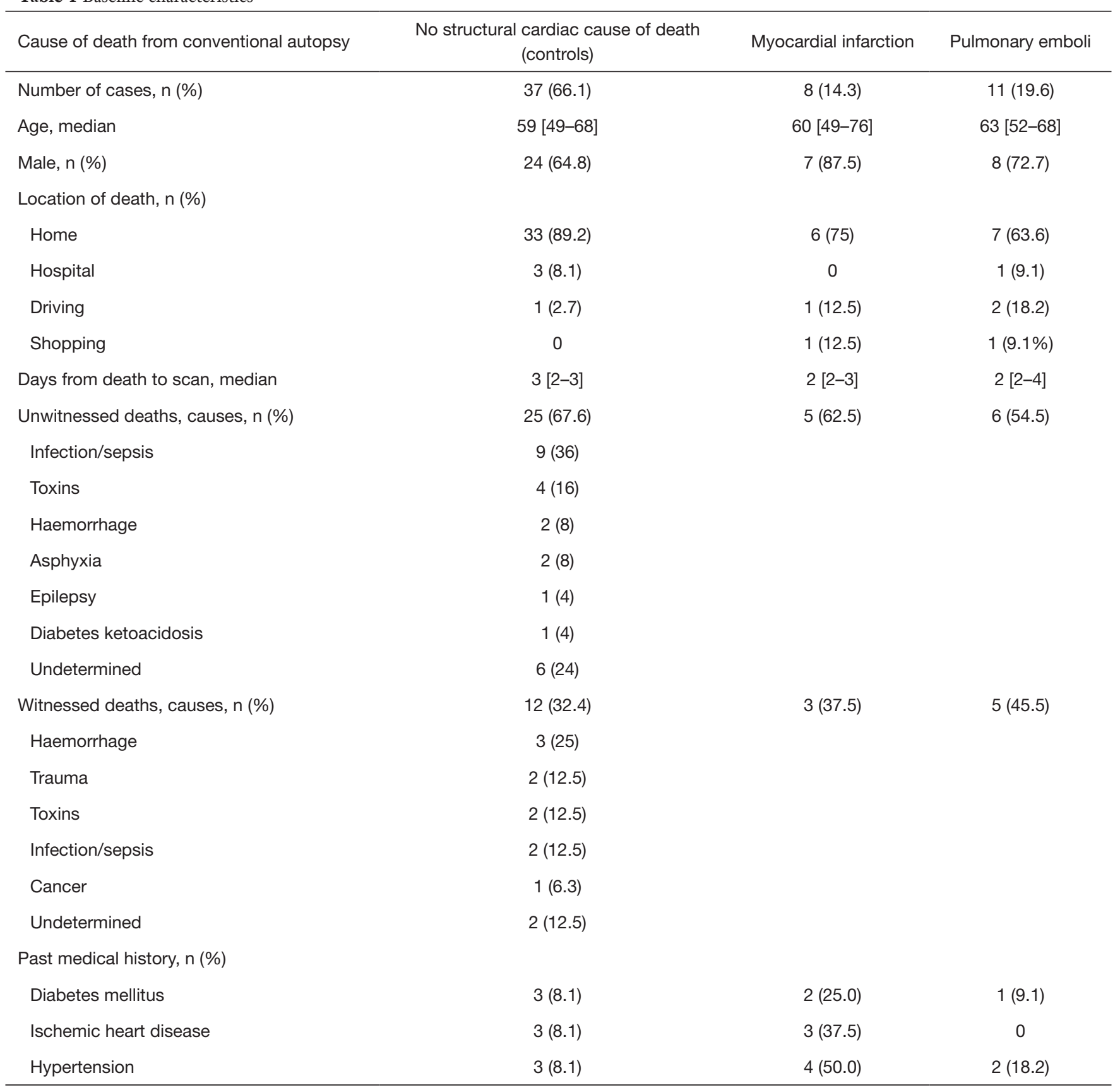

while admitted in hospital (two had sepsis, one died from pulmonary emboli following complex surgery and one had no identified cause of death), four (7.1\%) collapsed and died while driving a car or bike and two $(3.6 \%)$ collapsed and died while shopping; Table 1.

\section{Reproducibility}

The distribution of myocardial wall thickness, myocardial signal intensity and ventricular areas were Gaussian in all three groups. We were able to measure these parameters 
with a high degree of inter-observer reproducibility and intra-class correlation coefficient equal to $0.86[95 \%$ confidence interval (CI), 0.73-0.93; $\mathrm{P}<0.001]$. For the three parameters, there was a slight bias $0.86 \pm 1.68,1.6 \pm 6.8$ and $0.09 \pm 0.22$ but no tendency and only $2 / 56,1 / 56$ and $2 / 56$ values were slightly outside the limits of agreement. The intra-class correlation coefficient was strong for $(0.92$ with $95 \%$ CI, $0.81-0.96,0.92$ with $95 \%$ CI, $0.82-0.96$ and 0.86 with $95 \% \mathrm{CI}, 0.73-0.93)$.

\section{Left ventricular wall thickness}

In all three groups, the difference between the CMR and autopsy measured LV septal wall $\{14$ [13-16] vs. 16 [13-18], $\mathrm{P}=0.16\}$ and lateral wall thickness $\{15$ [13-17] vs. 16 [12-17], $\mathrm{P}=0.43\}$ was not statistically different but thicker than published ant-mortem values (10). In fact, in those with no structural cardiac abnormality, 37.8\% (14/37) would satisfy ante-mortem CMR criteria for HCM (wall thickness $\geq 15 \mathrm{~mm}$ ) despite none of them having evidence of myocyte disarray on autopsy examination $(15,16)$. In those with acute or subacute myocardial infarction, the median $\mathrm{LV}$ wall thickness of infarcted myocardial wall was $13 \mathrm{~mm}$ [12-13]. Additionally, there was no difference in wall thickness between those with or without hypertension; $\mathrm{P}=0.418$ and 0.691 .

The median CMR septal and lateral walls were significantly thicker for males compared to females but there was no difference between the CMR and autopsy measured $\mathrm{LV}$ wall measurements. The septal wall thickness was $15 \mathrm{~m} \mathrm{~m} \mathrm{[13-17]} \mathrm{for} \mathrm{males} \mathrm{and} 13 \mathrm{~mm}$ [12-13] for females $(\mathrm{P}=0.037)$ and the lateral wall was $16 \mathrm{~mm}$ [14-18] for males and $13 \mathrm{~mm}$ [12-15] for females $(\mathrm{P}=0.018)$.

\section{Myocardial tissue signal intensity}

In all three groups, non-infarcted septal walls had higher myocardial signal intensity than non-infarcted lateral walls $\{141 \mathrm{SI}[111-171]$ vs. $123 \mathrm{SI}[100-158], \mathrm{P}=0.093\}$ and greater signal indexed to skeletal muscle $\{1.9[1.8-2.4]$ vs. 1.7 [1.62.1], $\mathrm{P}=0.085\}$ but the difference was not significant. There was no statistical difference in the indexed signal intensity of the septal and lateral walls between males and females $(\mathrm{P}=0.081$ and 0.097$)$.

In those diagnosed with acute or subacute myocardial infarction on conventional autopsy, the corresponding region on CMR had hyper-intense signal intensity on T2 images (indexed to skeletal muscle) compared to normal myocardium in patients with no structural cardiac abnormality or pulmonary emboli $\{2.5[2.3-3.0]$ vs. 1.9 [1.8-2.5], $\mathrm{P}<0.01$; Figure 1 and Table 2\}. None of the other patients had areas of hyper-intensity. Using receiver operator characteristics (ROC) curve analysis, an indexed signal intensity $\geq 2.3$ had an area under the curve (AUC) of $0.83 ; 95 \%$ CI, 0.70 to 0.95 with a sensitivity of $89 \%$ and specificity of $68 \%$ for identifying subacute myocardial infarction in the post-mortem setting.

\section{Right and left ventricular area assessment}

In those who died with no structural cardiac abnormality or acute/subacute myocardial infarction, there was no statistical difference in median $\mathrm{RV}$ and $\mathrm{LV}$ cavity areas (indexed to body surface area); 6.0 [4.9-7.5] vs. $6.3 \mathrm{~cm}^{2} / \mathrm{m}^{2}$ [5.7-6.5] and $3.6 \mathrm{~cm}^{2} / \mathrm{m}^{2}$ [2.9-4.1] vs. $3.5 \mathrm{~cm}^{2} / \mathrm{m}^{2}$ [3.1-3.6], $\mathrm{P}=0.619$ and $\mathrm{P}=0.606$, respectively. In these two groups, the RV to $L V$ area ratio was 1.8 [1.5-2.0] and 1.9 [1.6-2.1], $\mathrm{P}=0.662$. There was no significant difference in $\mathrm{RV}, \mathrm{LV}$ area or $\mathrm{RV}$ to $\mathrm{LV}$ area ratio between males and females. $\mathrm{P}=0.381$, 0.812 and 0.102 .

In those who died from pulmonary emboli, the RV to $\mathrm{LV}$ area ratio were significantly higher than those who died with no structural cardiac abnormality or acute/subacute myocardial infarction $\{2.9$ [2.1-3.0] vs. 1.8 [1.4-2.0], $\mathrm{P}<0.01$; Figure 2 and Table 3\}. Using ROC curve analysis, a RV to $L V$ area ratio $\geq 2.3$ had an AUC of 0.931 ; CI, 0.85-1.00 with a sensitivity $85 \%$ and a specificity of $84 \%$ for identifying pulmonary emboli.

\section{Discussion}

Post-mortem CMR is a non-invasive alternative in the investigation of coronial deaths where conventional autopsy cannot be performed (6). In this pilot study, we correlate conventional autopsy findings to CMR imaging and assess post-mortem CMR parameters such as $L V$ wall thickness, myocardial signal intensity and ventricular cavity area. The major findings of this study are: (I) In the post-mortem setting, myocardial wall thickness is not reliable to diagnose hypertrophic cardiomyopathy without histological and/or genetic assessment. (II) Autopsy proven acute or subacute myocardial infarction can be suspected by an indexed (to skeletal muscle) myocardial signal intensity ratio $\geq 2.3$. (III) Autopsy proven pulmonary emboli can be suspected on CMR by an indexed $R V$ to $L V$ area ratio $\geq 2.3$.

During the post-mortem process, bodies are exposed 

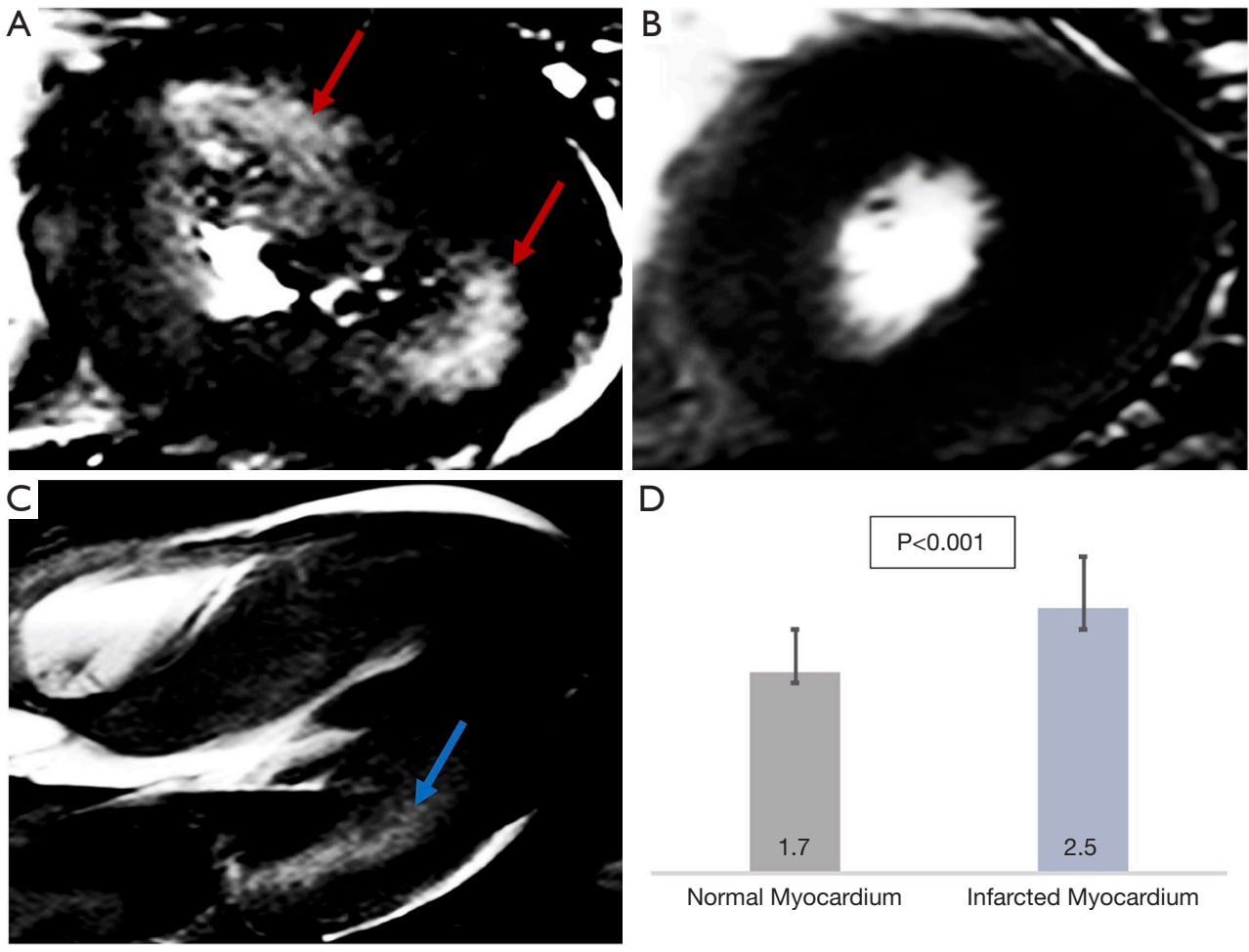

Figure 1 (A) Mid left ventricular (LV) short-axis T2 STIR cardiac magnetic resonance (CMR) image of patient with hyper-intense signal in the antero-septum and posterolateral region corresponding to 2 regions of myocardial infarction identified on conventional autopsy (red arrows); (B) Mid LV short-axis T2 STIR CMR image of patient without myocardial infarction or i.e., "normal heart"; (C) LV long axis T2STIR image of patient with hyper-intense signal in the inferoposterior region corresponding to region of myocardial infarction identified on conventional autopsy (blue arrow); (D) Median signal intensity ratio of normal myocardium normalised to skeletal muscle in patients without myocardial infarction or cardiac disease and hyper-intense myocardium normalised to deltoid muscle in patients with subacute myocardial infarction. STIR, short tau inversion recovery; CMR, cardiac magnetic resonance; LV, left ventricular; RV, right ventricular; LVOT, left ventricular outflow tract.

to cold temperatures and putrefaction that can change the structure and composition of organs. As a result, it can be difficult to identify pathological abnormalities on postmortem MR and accurately diagnose some cardiovascular conditions. In a group without cardiovascular disease or histological evidence of hypertrophic cardiomyopathy on autopsy, we found that the septal and lateral walls were thicker than ante-mortem measurements in comparable patients (15). Supporting this finding, a previous study compared ante-mortem and post-mortem cardiac CT scans and found that myocardial wall thickness was increased on post-mortem images even in those without cardiovascular disease (16). At present, there are no post-mortem imaging guidelines for HCM, making the diagnosis difficult without histological confirmation of myocyte disarray and/ or identifying known gene mutations. Moving forward, dedicated post-mortem cut-off values will need to be validated to improve the accuracy of the CMR imaging in this setting.

Myocardial infarction is a common cause of unexplained death but an accurate diagnosis with post-mortem CMR is difficult due to the poor resolution of coronary arteries and the inability to deliver contrast. Although criteria for identifying infarcted myocardium on post-mortem CMR without gadolinium has not been established, it can be visualised by $\mathrm{T} 2$-weighted $\mathrm{MR}$ sequences $(17,18)$. In fact, one study found that autopsy identified subacute myocardial infarction showed increased signal intensity on T2-weighted STIR and fluid-attenuated inverse recovery (FLAIR) imaging (17). In our cohort, we found that patients with autopsy confirmed acute/subacute myocardial infarction had increased T2-weighted signal intensity (indexed to skeletal muscle) in corresponding CMR areas. Specifically, we found that an indexed signal intensity ratio of $\geq 2.3$ was accurate 
Table 2 Characteristics and imaging parameters of individuals with myocardial infarction compared to individuals who died from other causes

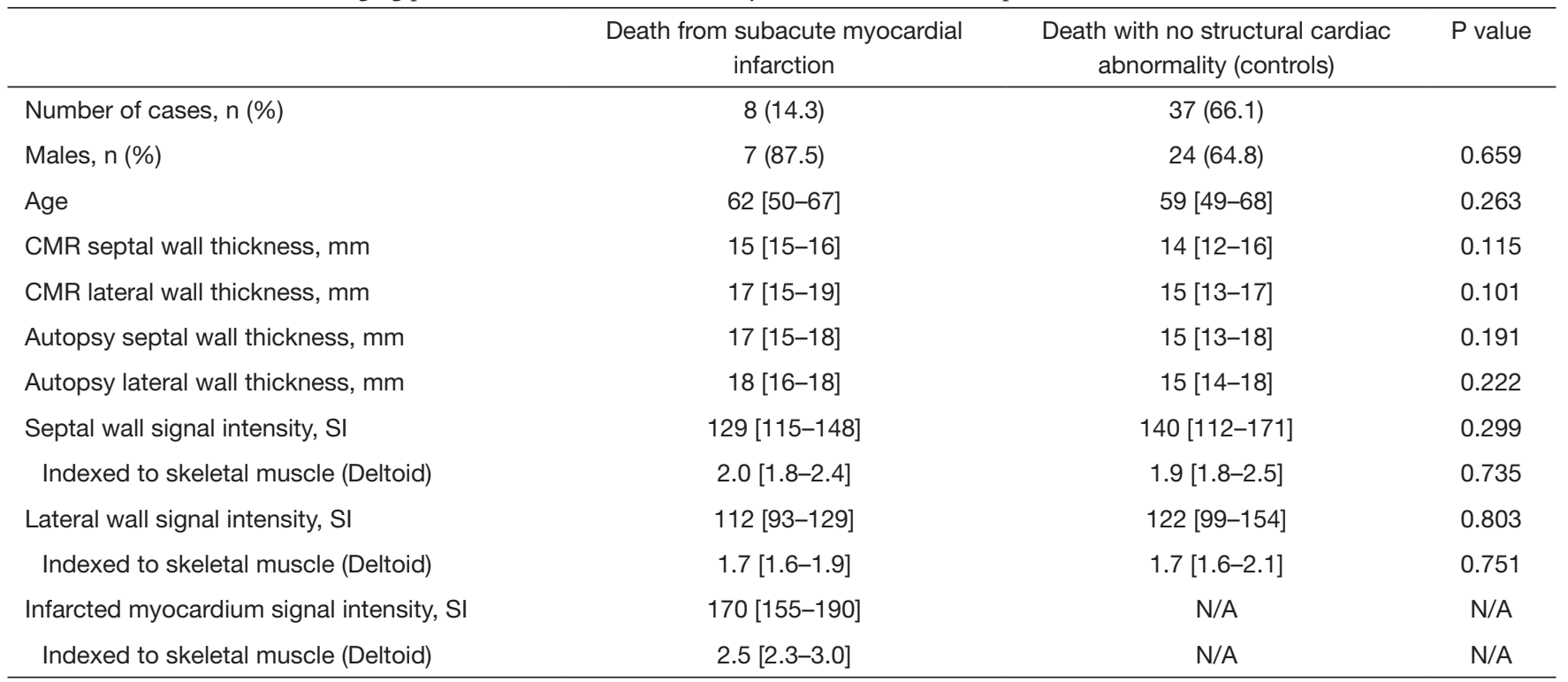
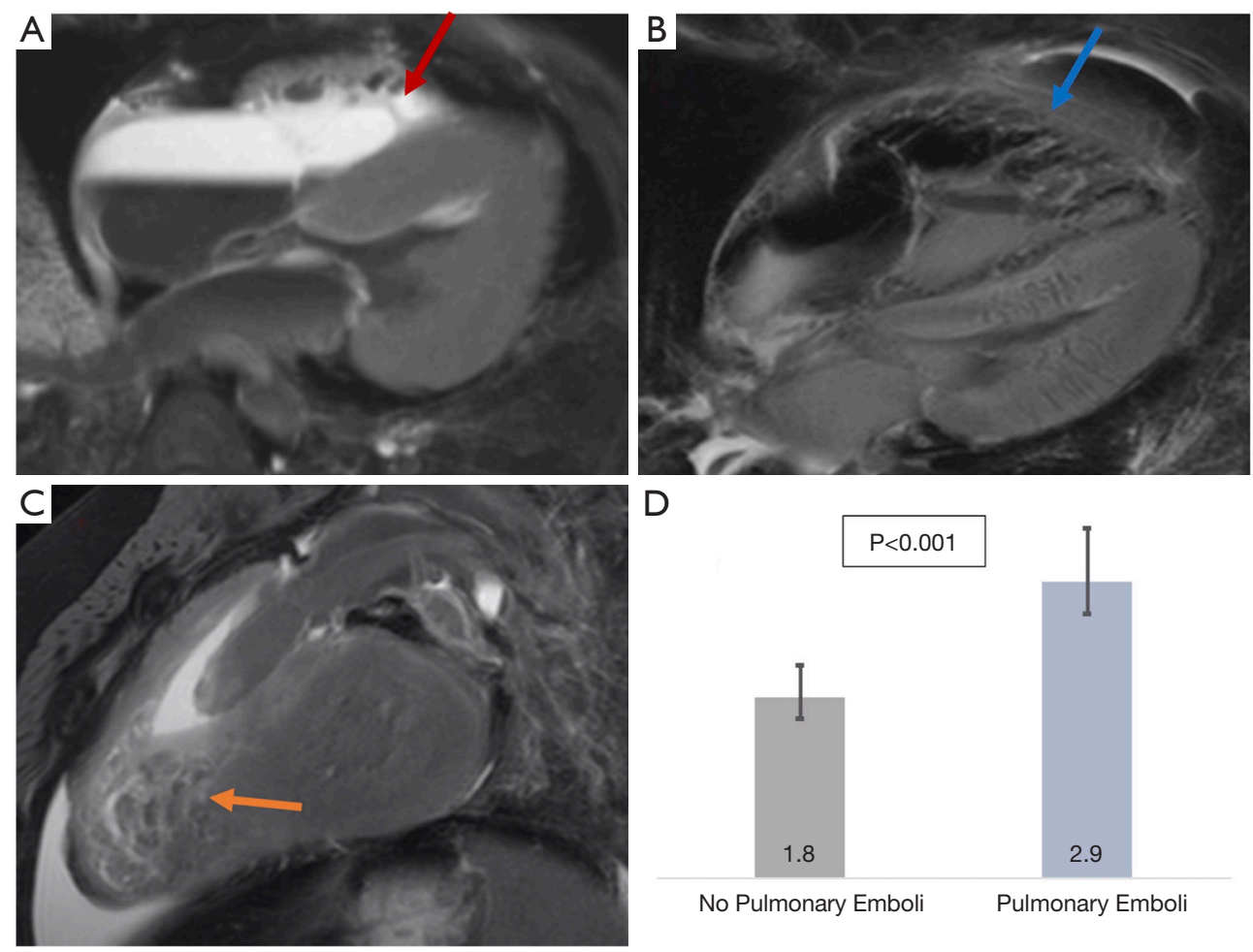

Figure 2 (A) Four-chamber T2 TSE cardiac magnetic resonance (CMR) image of patient without pulmonary emboli showing normal right ventricular (RV) cavity area (red arrow); (B) Four-chamber T2 TSE CMR image of a patient with autopsy confirmed pulmonary emboli showing dilated RV cavity area (blue arrow); (C) Right-ventricular outflow tract (RVOT) T2 TSE CMR image of dilated RV in patient with autopsy confirmed pulmonary emboli (orange arrow); (D) Median RV to LV volume ratio of patients with no pulmonary emboli and patients with pulmonary emboli. TSE, turbo spin echo; CMR, cardiac magnetic resonance; RV, right ventricular; LV, left ventricular; RVOT, right ventricular outflow. 
Table 3 Characteristics and imaging parameters of individuals with pulmonary emboli compared to individuals who died from other causes

\begin{tabular}{|c|c|c|c|}
\hline & $\begin{array}{l}\text { Death from pulmonary } \\
\text { emboli }\end{array}$ & $\begin{array}{l}\text { Death with no structural cardiac abnormality } \\
\text { (controls) }\end{array}$ & $P$ value \\
\hline Number of cases, $\mathrm{n}(\%)$ & $11(19.6)$ & $37(66.1)$ & \\
\hline Males, n (\%) & $8(73)$ & $24(64.8)$ & 1.000 \\
\hline Age & $64[34-66]$ & 59 [49-68] & 0.233 \\
\hline Autopsy septal wall thickness, $\mathrm{mm}$ & $15[15-16]$ & $15[13-18]$ & 0.828 \\
\hline Autopsy lateral wall thickness, $\mathrm{mm}$ & 15 [12-15] & $15[14-18]$ & 0.799 \\
\hline Septal wall signal intensity, SI & $143[119-151]$ & $140[112-171]$ & \\
\hline Indexed RV area, $\mathrm{cm}^{2} / \mathrm{m}^{2}$ & $7.9[5.6-9.3]$ & $6.0[4.9-7.5]$ & $0.041^{*}$ \\
\hline Indexed LV area, $\mathrm{cm}^{2} / \mathrm{m}^{2}$ & $2.7[2.0-3.7]$ & $3.6[2.9-4.1]$ & 0.333 \\
\hline $\mathrm{RV}$ to $\mathrm{LV}$ area ratio & $2.9[2.1-3.0]$ & $1.8[1.4-2.0]$ & $<0.001^{*}$ \\
\hline
\end{tabular}

${ }^{*} \mathrm{P}<0.05$.

in identifying acute/subacute myocardial infarction. To date, there is a paucity of data reporting myocardial signal alterations such as occurs with myocarditis on post-mortem CMR, but potential differential diagnoses need to be addressed in specific studies to define their post-mortem imaging appearance.

From studies examining the accuracy of post-mortem CMR, pulmonary thrombus was often missed or incorrectly diagnosed largely due to the presence of post-mortem clot (7). As such, identifying pulmonary emboli in the postmortem setting remains challenging. In individuals with autopsy confirmed pulmonary emboli, we found definite $\mathrm{RV}$ enlargement on CMR with an indexed $\mathrm{RV}$ to $\mathrm{LV}$ area ratio $>2.3$. In support of this finding, an ante-mortem study found that an RV to $\mathrm{LV}$ area ratio on cardiac CT was accurate at identifying pulmonary emboli and assessing clinical severity (19). Therefore, measuring ventricular area ratios can be a useful marker to indicate $\mathrm{RV}$ and/or pulmonary artery pathology in patients with unexplained death who cannot undergo autopsy. It is acknowledged that these changes may be seen in other RV pathological conditions, where the numbers in our cohort were too small to make robust conclusions but do warrant further investigation.
The results of this pilot study are limited by a single centre involvement and a relatively small number of scans. As such, we suggest that further validation of our proposed CMR parameters be performed in larger studies. Cases were selected from our original blinded study that demonstrated the sensitivity and specificity of post-mortem CMR to identify causes of death (7); however, for this study, the investigators were aware of the results of the autopsy and CMR studies. Ultimately, this may be a potential confounding variable. That said, we felt that evaluating CMR parameters directly against the histopathologic "gold standard" i.e., autopsy would maximize accuracy of our results. The individuals referred for coronial investigation were mainly Caucasoid and therefore the parameters may not be generalisable to other ethnic groups. Similar to ante-mortem MR, post-mortem imaging can identify the coronary arterial course but it does not have the resolution to identify obstructive coronary artery disease. As such, myocardial infarction can only be recognised by examining "downstream" effects within the myocardium. At the time of the study, we did not have access to T1 mapping sequences, but we accept evaluating changes with these sequences may provide additional information. Ventricular hypertrophy was reported with reference to published 
guidelines; however, the final diagnosis was decided by the pathologist and therefore may not be standardized. Three patients in the control group had a history of diabetes mellitus, ischemic heart disease and/or hypertension but the cause of death was non-cardiovascular (trauma, sepsis and toxins). Although there was non-specific fibrosis on histological examination, there was no evidence of structural heart disease or acute/subacute myocardial infarction. Never the less, this may be a confounding factor and needs to be considered. Finally, the time interval from death to post-mortem imaging/examination in our study was relatively short. However, there is uncertainty about the validity of our proposed diagnostic parameters with longer time intervals and more advanced body decomposition,

\section{Conclusions}

In this pilot study, we present preliminary data that suggests potential post-mortem CMR parameters for identifying important cardiovascular abnormalities. Measuring LV thickness with CMR alone is not reliable in diagnosing HCM but elevated T2 signal intensity can be used as a marker for acute or subacute myocardial infarction and increased $\mathrm{RV}$ to $\mathrm{LV}$ area ratios can help identify pulmonary emboli. Larger studies are required to confirm dedicated CMR parameters and enhance the reporting accuracy of post-mortem imaging.

\section{Acknowledgments}

Funding: The study was conducted with the permission of the Office of the South Australia State Coroners and performed with maintenance of confidentiality in accordance with NHMRC human ethics guidelines. The State Coroner for South Australia approved a joint initiative between Forensic Science South Australia and the South Australian Health and Medical Research Institute (SAHMRI) to trial post-mortem post-mortem magnetic resonance and computed tomography imaging of coronial cases using funding provided by the South Australian Government.

\section{Footnote}

Reporting Checklist: The authors have completed the STROBE reporting checklist. Available at: http://dx.doi. org/10.21037/cdt-20-948
Peer Review File: Available at: http://dx.doi.org/10.21037/ cdt-20-948

Data Sharing Statement: Available at: http://dx.doi. org/10.21037/cdt-20-948

Conflicts of Interest: All authors have completed the ICMJE uniform disclosure form (available at: http://dx.doi. org/10.21037/cdt-20-948). The authors have no conflicts of interest to declare.

Ethical Statement: The authors are accountable for all aspects of the work in ensuring that questions related to the accuracy or integrity of any part of the work are appropriately investigated and resolved. The study was conducted in accordance with the Declaration of Helsinki (as revised in 2013). The State Coroner for South Australia approved a joint initiative between Forensic Science South Australia and the South Australian Health and Medical Research Institute (SAHMRI) and performed in accordance with NHMRC human ethics guidelines and informed consent was taken from all the patients.

Open Access Statement: This is an Open Access article distributed in accordance with the Creative Commons Attribution-NonCommercial-NoDerivs 4.0 International License (CC BY-NC-ND 4.0), which permits the noncommercial replication and distribution of the article with the strict proviso that no changes or edits are made and the original work is properly cited (including links to both the formal publication through the relevant DOI and the license). See: https://creativecommons.org/licenses/by-nc-nd/4.0/.

\section{References}

1. Royal College of Pathologists of Australasia Autopsy Working Party. The decline of the hospital autopsy: a safety and quality issue for healthcare in Australia. Med J Australia 2004;180:281-5.

2. Loughrey MB, McCluggage WG, Toner PG. The declining autopsy rate and clinicians' attitudes. Ulster Med J 2000;69:83-9.

3. Puranik R, Gray B, Lackey H, et al. Comparison of conventional autopsy and magnetic resonance imaging in determining the cause of sudden death in the young. $\mathrm{J}$ Cardiovasc Magn Reson 2014;16:44.

4. Taylor AM, Sebire NJ, Ashworth MT, et al. Postmortem cardiovascular magnetic resonance imaging in fetuses and 
children. Circulation 2014;129:1937-44.

5. Femia G, Semsarian C, Langlois N, et al. Postmortem imaging adjudicated sudden death: causes and controversies. Heart Lung Circ 2019;28:15-21.

6. Roberts IS, Benamore RE, Benbow EW, et al. Postmortem imaging as an alternatie to autopsy in the diagnosis of adult death: a validaion study. Lancet 2012;379:136-42.

7. Femia G, Langlois N, Raleigh J, et al. Comparing conventional autopsy to post-mortem MR and CT in determining the cause of sudden and/or unexplained death. Forensic Sci Med Pathol 2021;17:10-8.

8. Kitzman DW, Scholz DJ, Hagen PT, et al. Age related changes in normal human hearts during the first 10 decades of life. Part II (Maturity): A quantitative anatomic study of 765 specimens from subjects 20 to 99 years old. Mayo Clin Proc 1988;63:137-46.

9. Gaitskell K, Perera R, Sollieux E. Derivation of new reference tables for human heart weights in light of increasing body mass index. J Clin Pathol 2011;64:358-62.

10. Sheppard MN. Approach to the cardiac autopsy. J Clin Pathol 2012;65:484-95.

11. Cunningham KS, Spears DA, Care M. Evaluation of cardiac hypertrophy in the setting of sudden cardiac death. Forensic Sci Res 2019;4:223-40.

12. Michaud K, Basso C, d'Amati G, et al. Diagnosis of myocardial infarction at autopsy: AECVP reappraisal in the light of the current clinical classification. Virchows
Arch 2020;476:179-94.

13. Shrout PE, Fleiss JL. Intraclass correlation: uses in assessing rater reliability. Psychol Bull 1979;86:420-8.

14. Hanley JA, McNeil BJ. A method of comparing the areas under receiver operating characteristic curves derived from the same cases. Radiology 1983;148:839-43.

15. Kawel-Boehm N, Maceira A, Valsangiacomo-Buechel E, et al. Normal values for cardiovascular magnetic resonance in adults and children. J Cardiovasc Magn Reson 2015;17:29.

16. Okuma $\mathrm{H}$, Gonol $W$, Ishida $M$, et al. Heart wall is thicker on postmortem computed tomography than on ante mortem computed tomography: the first longitudinal study. PLoS One 2013;8:e76026.

17. Jackowski C, Christe A, Sonnenschein M, et al. Postmortem unenhanced magnetic resonance imaging of myocardial infarction in correlation to histological infarction age characterization. Eur Heart J 2006;27:2459-67.

18. Ruder TD, Ebert LC, Khattab A, et al. Edema is a sign of early acute myocardial infarction on post-mortem magnetic resonance imaging. Forensic Sci Med Pathol 2013;9:501-5.

19. Ghaye B, Ghuysen A, Bruyere P, et al. Can CT pulmonary angiography allow assessment of severity and prognosis in patients presenting with pulmonary embolism? What the radiologist needs to know. Radiographics 2006;26:23-9.

Cite this article as: Femia G, Langlois N, Raleigh J, Perumal SR, Semsarian C, Puranik R. Post-mortem cardiac magnetic resonance parameters in normal and diseased conditions. Cardiovasc Diagn Ther 2021;11(2):373-382. doi: 10.21037/cdt20-948 